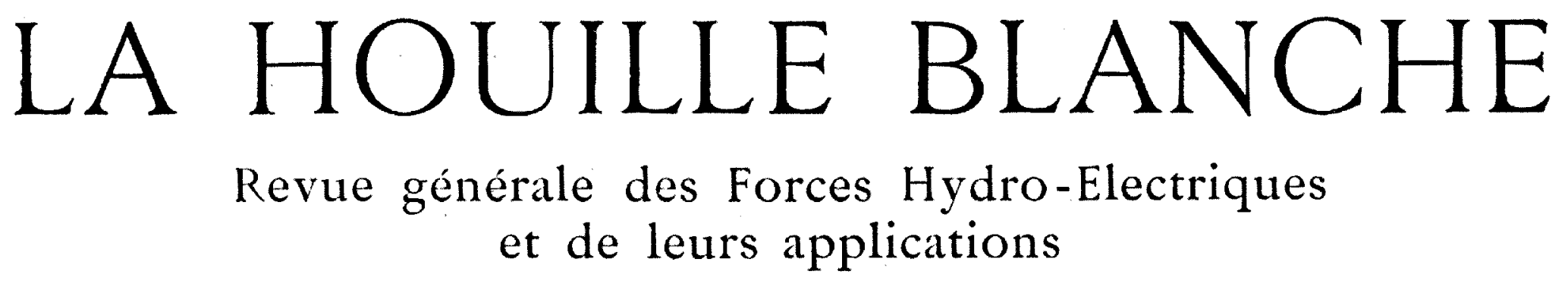

6 e Année. - Août 1907. - No 8.

La Houille noire a fait l'Industrie moderne; la Houille blanche la transformera.

\section{LA HOULLLE BLANCHE ET LA QUESTION SYLVO-PASTORALE}

Confórence faite aux élèves de l'Institut Electrotechnique de Gronoble, par notre collaborateur, le Commandant Aubebrand, sius les auspices al la Societe pour le diveloppement de l'Enseignement technique près l' Lniversité de Grenoble.

(Suile el fin)

Certains peut-êtrc, prenant texte de ce qui vient d'advenir aux viticulteurs du Midi que la mine vient d'atteindre pour des raisons multiples, parmi lesquelles on fait valoir l'exclusivisme avec lequel ils se sont adonnés uniquement à Ia culture de la vigne, nous accuserons de pousser les montagnards à un désastre pareil en leur conseillant des cultures trop uniformes. Nous devons prévoir ce reproche et le combattre avant même qu'il ne se formule.

Disons tout d'abord qu'il ne faut comparer que les choses comparatiles et que la position du montagnard est toute différente do celle du vigneron languedocien. Sans doute dans le Languedoc il $y$ a nombre de terres qui ne sont bonnos quà la vigne, mais nombre d'autres aussi qui auraient pu porter des prés et des céréales ont été inconsidérément converlies en vignobles : or, celte cenversion ne s'est faite que sous l'empire de l'appat du lucre tt non sous la pression dune nécessité de salut public ; dans la montagne il $\mathrm{y}$ a, de toute évidence, le pilus grand intêrêt public à cesser les labours et à laisser lherbe et le bois envahir des terrains jadis essartés et labourés. Ia situation est donc toute différente ici et là el c'est à cela qu'il faut bien prendre garde.

De ce que nous avons dit qu'il ne fallait dans la montagne faire que du pré et du bois, el non des labours et des défoncements il ne suit pas d'abord qu'il no puisso tout de même y avoír place près de la maison pour un jardin où la famille trouvera ses aliments végétaux. C'est de la très grande majorité des terres que nous parlons des $9 / 10$ par exemple ; mais il semble bien qu'il sera toujours possible de trouver $1 / 10$ des terrains en position convenable sur des replats pour y pratiquer quelques cultures maraîcheres sans pour cela faire naître le moindre danger.

Le montagnard, au surplus, ne sera pas dans la position où le Languedocien s'est mis de gajeté de cœur, s'il sait garder outre son indispensable jardinet, ses ruches et ses arbres a fruits. Les cueilleltes variées sont pour lui une ressource estimable, et parmi tous les fruits que la nature lui donne presque sans soins, comme pour le dédommager de la rudesse de son habital, la chảtaigne principalement, déve. loppée sur les terrains primitifs, est une ressource de haute valeur pour le commerce, l'alimentation de l'homme et des animaux qu'il emploie. Les conditions naturelles ne sont done et ne seront done jamais les mêmes à la montagne que dans les vignobles trop doveloppés, si l'homme se contente de suivre les indications de la nature.

Il est vrai que le montagnard est aussi cupide que le vigne ron et que l'appat du lucre immédiat el rapide le sollicite aussi impérieusement. Le châtaignier, dont nous venons de parler est désormais très menacé en France el en Corse par l'industrie toute récente de lextrait de chataignier ou liqueur de tannin à l'usage des tanneurs. Cel extrail permel, en effet, de donner un bel aspect aux peaux, mais il ny a pas que le châtaignier qui soil riche en tannin, le chène, le saule, le bouleau, le noyer et d'autres encore, en recèlent de notables quantités et pourraient donner la liqueur. Ce qui scmble avoir fait jeter le dévolu des chercheurs sur le chalaignicr, c'est une maladie qui en a lait périr beaucoup (le pied noir) et qui a poussé les gens à tirer le meilleur parti d'un arbre qui dépérissait. Malheureusement la mesure a été dépassée et il faut maintenant songer a prendre des mesures conservatrices dc ce qui nous reste, el icstauratrices de ce qui est parti.

Nous done, qui voulons que le montagnard use de la parure végétale du sol en bon père de famille, nous sommes Join, on le voit, de le pousser a un exclusivisme irraisonné et ruineux.

Ja montagne laissée à elle-mème produit naturellement de la glace, de l'herbe, du bois et de l'eau.

Lindustrie de la houille blanche donne un débouché économique inattendu à ses forces nićcaniques, soit qu'on les utilise dans la montagne même en ceuvres diverses, mais principalement à l'électro-chimie et à l'électro-métallurgie, soit qu'on la transporle au loin pour actionner mille machines altelées dans les plaines à des métiers divers.

Toutes vos études ici ont été orientées vers ces ulilisations, et je m'en voudrais d'insister trop longuement sur cel objet.

Jusque dans ces derniers temps encore, la difficulté d'exporter au loin la glace des glaciers, avait empeché de s'en servir pour toutes les réfrigérations industrielles ou ménagères. Maintenant, le développement du réseau ferré se condé aussi par l'emploi de câbles el de transporteurs pour l'exploitation directe de la carrière de glace, met quelquesunes de celles-ci à proximité suffisante des lieux de consommation pour que celle industrie devienne dans certain cas rémunératrice, aussi avons nous $v u$, il $y$ a déja plus de quinze ans, certains glaciers des $A$ lpes exploites de cetle facon.

C'est là une industrie fort peu recommandable et qui aurait du, nous semble-t.il, être écartée tout d'abord par une sorte de question préalable facile à motiver.

Dabord nos glaciers, regulateurs de beaucoup de nos torrents alpins, sont en régression, ainsi que les constatations des annees dernières l'ont montré comme vous le savez. Il semble donc qu'il $\mathrm{y}$ ait une raison d'interêt supérieur, sinon général ou de salut public, à en interdire lo débit en tranches.

Il faut ajouter que, malgré la rarefaction relatlve des microorganismes dans les haules régions de l'a'mosphère, les glaces qui proviennent de ces amas ne sont pas stériles de germes, et qu'elles en collectent aussi en voyageant. Ces glaces, encore que moins dangereuses pour la consommation alimentaire que celles qu'on peut recueillir en hiver a 12 surface des étangs, lacs, cours d'eau, et conserver en gla : 
cière, est cependant dangereuse et doit élre jéservée à la production indirecte du froid. Pour ces raisons encore, l'Ltat fui s'est improvisé nolve luleur hygiénique, devrait en intexdire l'exploitation industrielie.

Ft ces-interdictions seraient dautant plus freiles a prononcer que, chez nous, airsi que la démontré $M$. Ed. Thureau, dans le très intéressant mémoire inséré par lui, en 1879, dans liAnnuaire du Club Alpin Francais, les glaciers sont du domaine prive de ilitat. Leur exploitation devait done laire l'objet de toules les formalités dont celuici envirume ses concessions, el si jamais obstruction ou interdiction ont bte opportuncs, cest bicn dans ce cas. Ur, nous crovons qua ren na été fait dans ce sens. (noique persorindlement, nous soyons très neltement opposé à l'ingérenec de letat dans les questions industrielles, nous niresitons pas a prendre posilion jei cn laveur de l'interdiction absolue. Vous voyez pourutuoi.

Cette industrie, au surplus, n'a pas d'avenir, la frigorifica. lion artilieielle qui permet d'obtenir, à fort bas prix, les réfrisirations les plus varites, sur les denrees memes, et de cristalliser des eaux absolunient pures pour produile des slaces de table, ne lui permelur pás de se développer. Mais, (n) attendant quils se ruinent eux-mimes, les exptoilants du glacier ont le temps de rumer celui-ci et c'est ce que le louvoil he doit pas permettre. Son opposition sera la une applicalion toute normale de ses obligations de police supérieure, c'est son droit el son devoir absolas.

Les industries liées a lexploitation des focets sont bien autrement nombreuses et inforessantes.

La forêt donne du bois de chaulage, même encore du charbon, olle donne du bois d'auve, du bois a denbres: pour en faire de la celhulose da décontiquer pour en tirer ifu tannin, des produits de distiblalion, acide acótique, ascool, goudron, du liège, des écorces, elc.

La collulose, vous le sive\%, est le fondement de la pâte à papier, du cothioüd el de tons ses succotanes. lost sous los ivalars les plus divers, un produit indispensable a ia vie noderne.

Cette facilibe déconlement du produit est un danger meragant pour la ford. Il a remplace, avec agoravalion, le danger dautrefois qu'ctat la métaldrgie au bois. Nombre de forels onl jadis blé devorées par les foyers el les foumeanx de ros aicux, il est a craindre que la diffusion de la presse n'ait pour les nations modernes, la conséquence de leur cnlever lents bois pour les daire mànor par les defibreuses u digérer dans les piles de procteries el les euves électrolytiques de blanehissage, avant de les laminer dans les rolatives. Sclon l'importance de son tirage, un quotidien consomme par jour de 2 a 200 arbres, ages de cinquanle ans, of lous le: jours il nait des joumnux et des hives nouveaux.

$1 \mathrm{y}$ y arait heu, dans cet ordye d'idées, de suivre avec une attention soutenue, les essais que les Américains ont fail lannée dernicre et ont du poursuive depuis, avec la suite qui caractérise leurs idées, de fabriquer du papier avec la lige du colonnier. Nous poursuivons méthodifuement, sous l'influence d'une association cotonnière nationale, la complantadion méthodique du colomier au Sénégal et dans toules les parties de notre Afrigue necilentate qui se pretent it ce sence de cuture. Il pourail y avoir la un moven indirect d'émargner nos arbres, tout en donnant ì la presse son aliment indispensable. Qu'on songe en effel, qu'wn sapin (le 5 ans, cube en moycune $1 \mathrm{~m}^{3}$ de bois se traduisant par $500 \mathrm{kilog}$. de pate sèche: qu'bl faut donc 2 de ces arbres pour une tonne de papier, et quà lallure à laquedle on fait jouer les presses sur be monde entier; it est urgent de trouver des succédanes du bois.

Déjà le danger paraît assez menaçant pour ru'on songe aux moyens de l'enrayer. I semble que le jeu de la loi commerciale dio liorre é de la demande, puisse étre là dune réelle efferciśs, mais il peut y aroir beacoute de mal fait avant qu'elle at à intepvenir.
Plus de la moitie des pays civilisés produisent actuellement moins de bois qu'il ne leur en laut et sont contraints d'en acheter à ceux gui en ont un excédent. Mais ceux-ci, euxmêmes, commencent à élre menaces, la dépense de leurs réserkes allant plus vite que leur reconstalution.

Nous reviendrons plus loin sur les remedes à apporter à cette situation.

Le pré produil le bélail et, là encore, l'homme agit plus en prodigue qu'en bon père de famille. Il mésuse de ses pâturages en y plaçant plus de betes quils nen peuvent nourrir et en $y$ laissant comme un droit de premption a celles qui sont le plus redoutables, les monions et les chèvres.

Cherchons-en les principales raisons.

La chevre qui coute envron vingl-cinq rancs d'achat, est un des animaux les plus productifs quon puisse exploiter. on lappelle avec juste raison la vache du pauvre; olle donne en abondance un lait fort nourrissant, dont on tire dicxcellents fromages. Sa dépouille donne de la viande, de la peau et des comes. Une chevre adnl te mange 2 kil. de foin par jour, uno vache en exige $10 \mathrm{kil}$. donc autant que cing chevros; malheu. reusement, en pâturage libre, la chèvre détruit tout ce qu'elle allaude. On le sait depuis longtemps. En août 1730 , le Parlement de Grenoble jntendisat par arret exprès, et sous la sanction de peines sèvères, d'en entrelenir aucune sans autorisation réservée aux seuls pauvres. Des manicipalités on suivi cet exemple, soib en prolabant lanimal purement et simplement, soit en lui imposant une capitalion élové, de $20 \%$ de sa valour vinale par exemple, done une somme voisine de $5 \mathrm{rr}$ par tete.

La chère est particulièrement redoutable aux jeunes pousses arbustives et aux bourgeons bas des arbres. Dressé sur ses palteo de deritere elle yeut, avec ses donts, facilement athejudre les pousses qui sont it $2^{\text {20 }}$ au-dessus du sol. 1] y a dene un intérét majeur à lécarter de lous los baliveaux qu nont pas encore franchement dipasse celle stature.

Après la chevre, le montonl. Celui-ci est moins dangereux, matis il lest encore beaucoup. Si on le mel en nombre raisonnable sur un pré, comme l'engrais qu'il produl est très riehe, il laméliore en le fumant énergiquoment, mais cela a une condition cependant, cest qu'il y trouve loujours de lherbe en sullsance de son appélit. Si celle-ci vient à se rarefter, comme il fatl bien gu'il mange, il s'athque aux racines, les déchansse et ruine le pré. Quand il en est ainsi, c'est qu'on a mis sur celle aire trop de bêtes, et le pretinement facilenent dépété de celles-ci sur un sol dont l'épldeme ost dejà blesso, entraine la pune du terain. Survienne un abirl d'eau, eelui-ci se ravine, se dissout dans la nappe lifuide epanduc et roule a labine en ravageant fout sur sor passage. Tous ceux gu ont parconru les montagnes, ont pu conslater gue nombre de ravins vavageurs, nont pas eu dature origine. In he faut donc pas trop nettre de montons sur un pré, ne pas dépasser sa possibilité eomme disent les forestiers.

Or, un mouton coùte unf trentaine de faracs, à peu près comme une chère, une vache cotle autant gu'une douzaine de moutons. Lo montagnard, trop pauve pour acheter une vache, pourra se procumrune chevre, ou deux, et une derndonzane de moutons, et ne pas perdre tout l'intêrèt de son argent.

De plus la vache qui contera plus dlachal coûtera aussi phus dentretien, et no pourna artiver a donner un produit réeltement remunérateur que si elle n'est pas a l'élat d'échantillon isole; lexportation du lait, la fabricalion du fromage et du beurre ne sont des operations réllement remuneratrices que si elles peuvent se faire stu de gresses masses, représentant des voleurs capables de supporter sans en être trop gravement smrehargées, le prix des fuis d'exploitation, de transport et de vente aux lieux habités par la cliertele.

Pour ces raisons, auxquelles il convient de joindre dans une certaine mesure, la différence dagilite de ces bestiaux, 
on hauve plutot des chèvres et des moutons que des vaches dans la haute montague.

ll y en a une aubre : la transhumance. La transhumance est lexplatation du pré montagneux par un moulon élrauger, mouton gascon pour les palurages des Causses :Cévomors et Quercynois, espagnal pour le paturage pyrénéen, provengal pour le pâturage alpin.

Tous les ans, a moment de la promière fonte des neiges, des hordes oximes remontent le Rhòne el les valtées affluentes, et par Inoupeaux de 1000 a 10000 têles, viement cstiver dans les pramics hautes de notre pays. Vous les avez tou pencontrés avec leurs bayles, leurs chiens, los ànes et les mulets de leurs conducteurs.

A pelites joumées, cette armée vorace qui vient s'engraisser dans nos montagnes, gagne les communaux où, moyennant un loyer annuel variant de 0 fr. 75 a 1 fr. par tete, versés dans la caisse manicipale, ils pour ront séjourner jusqu"a la première bise. C'est le rersement de $750 \mathrm{fr}$. à $10,000 \mathrm{fr}$. dans leurs caisses, qui incile les montagnards à hospitaliser ces intrus. Natureherrent, plas ils en accueblent, phus la recetic municipale est elevée; mais ausi plus vite et plus profondément la monlayne sabime.

Fous avons vu plus haut de quelle conséquence était sa pauveté pour le montagnard individuellement, la commune montagnarde est adéruate au inontagnard, olle est paure aussi et, comme tous les pauvres, ello préfere un liens à deux tu liauras, le produit differé semble un leurre dans ce milieu et le paiement au complant $y$ séduit plus les gens que le crédit ouvert sur l'avenir.

Cette attraction du profit immediat a dé lelle que, maintes 6ois, des bois-clairières, des prés-bois, des lisières de fonêts, des forêts mèmes, ont été brulés, anéantis, pour faire cles prés a domner a tondre aux transhumants.

Voila cui vous explique que, maigré les inconvénicnts tres evidents pour tous les yeux rue la transhumance emporte avec elle, il y ait encore des gens de la haute montagne pour lui trouver du mérite. Dans un moment, nous dirons très nettement lavenix que nous désirons tui voir faire, pour linstant continuons l'énumération sommaire des industrit. de la montassne.

Pour mémoire, nous devons signaler les mires de médaux et de combustibles, les carrières de pierres à bâlir, de plâtres et de ciments, encore que l'invention de Vical ait permis de créer ceux-ci partout où se trouve du calcaire et de largile.

Enfin, il y a une industrie que nous cherchons à implan ter dans nos montagnes, celle du tourisme. Elle procede de celte idée que les tableaux que la nature offre chez nous, ne sont pas inferieurs a ceux qu'elle offe dans les pays etrangers el que notre race avant le bon désir deetre accueillantr, rous pouvons altirer chez nous, on tout temps, bon nombre de visiteurs qui viendront nous enrichir.

Ce serait une erreur à des ingćnieurs de se désintéresser de ce cỏ lé de la question. D'abord, ils sont touristes a leurs heures, comme tout le monde maintenant, mais ils sont aussi les auxiliaires tout indiqués de la mise en couvre de notre patrimoine, pour y rendre la vie agróable et commode. Je ne serais pas en peine de vous produire une longue liste de localités qui ont fait installer la lumière électrique, ou des tramways, a l'intention des visiteurs qu'elles cherchent a atiorer.

Mais il y'a une autre face à la question. Il y a le coté esthétique ! C'est une préoccupation que vous devez désormais avoir au mème titre que celle de la solidité de vos ponts et du bon agencement de vos usines. Ia loi vous en fait une obligation professionnelle, non pas la loi d'avril 1906, qui régente la protection des sites et monuments pittoresques, mais la loi actuellement en élaboration, au sujet des usines hydrauliques.

Vous devez désormais faire entrer les considérations esthéticues dans vos projels.
Je n'insisterai pas sur ce poinl, il me suffl de vous le signaler pour que vous lui fassiez dans vos préoccurations professionnelles, la part et la mesure équilables qui lui l’eviennent. Ciest en ces matières que le bon sons est phus souverain que les lextes et les entranements.

Si, comme je lespère, l'énuméralion quasiment cinematographique, que je wiens de vous faire, vous a édines sur ha connexion des questions sylvo-pastomales, aveo les etudes plus techniques auxquelles vous vous deshnez, je pense quo vous ne vous refuserez pas à recomaîlre que sans le pri et ha fort, le barago est de nulle valeur, tandis que sans b barrage, la foret el le pré soul deja un puissant rigulateur et que, aidés par le barrage, ils sont un l'égulateur presque parfait.

Donc, tous les industriels tributaires, non seulement de lit houille blanche, mais encore de loules les induslries annexes que je vous ai énumérées, ont un intérêt direct daus les questions de prés, de bois el d'eaux, en monagne; mais encore les commerçants et les consommateurs. Nutant dire lout be monde $\mathrm{el}$ a bien prendre, octte formule elargie contiont. phus de vérité que loube fornule restreinle.

Au cours de cet expose, trop succinct, encore qu'il vous paraisse peut-être un peu long, nous avons pu constater que létal actuel n'etait pas exempl de monaces redoulables pour lavenir. Nous sommes fondes a craindre la diselle deau provenant de labus dans l'usage de nos bois ol de nos prés, nous sommes aussi tròs autorisós à nous natlor dun avenir séduisant si nous savons en temps opporlun, c'està-dire promptement, porter remide à la déforestation et à la ruine de nos paturages.

Nous entrevoyons qu'il $y$ a quelque chose à faire, mais quoi exactement el comment?

Ia première jdéc qui vienl en pareil cas à des civilisés, surtout à des Francais assouplis par une contralisation multiseculaire, c'ust de se retourner vors le Gouverncment, agent. de l'Etat, et d'implorer sa prolection el son aide. On ne s'en est pas lail faute et letat a ble sollicite de vingl manieres.

Malheureusement, ou heureusement peul-otre dans le cas actuel, nous avons déja chargí lWtal de lant ef tant de: hesognes, qu'il y succombe, qu'il tha ni assez dacrents, ni assez de finances pour moner à bout une telle entroprise.

La seconde idée est de charger jiflai, en verta de son pouvoir de haule police, de laire appliquer avec rigueur des lois dont on demande la promulgation, destinces a reprimel les errements fautirs dans lesquels persiste encore la masse ignorante.

Nous navons pas à disculer ici celle these. Disons seule. ment que nous avons des lois excellentes sur Ia matiere ; qu'elles aient besoin de quelques compléments pour les mettre au point, c'est possible; mais que, telles ruelles sonl, si elles étaient appliquées avec fermelé el sagosse, olles so montreraient tres efficaces. Ce gui entravo leur emploi, c'est, plus que leur insuffisance, l'élal de nos moeurs, l'ignoraneo du public, son indiférence, le manque d'inilialive des parliculiers, c'est surtout la qu'est le mal, el la premiere comme la plus décisive victoire a remporter, c'est de nous reformer sur ce point.

Pour notre compte, le présent cnlretien est un geste en co sens et je l'estimerais comme nous rapprochant sensiblement du but si vous sorticz d'ici convaincus de ce rulil y a à faire et résoius a vous y employer, dans la persuasion que ce sera pour vous laccompliscement de votre devoil professionnel, autant que peut letre un essai au frein ou to relevé d'une caractéristique de machine.

Il nous faut done aller a la monlame sans allendre qu'clls. vienne à nous, el instruire d'abord Je montagnard du danger que lui fait courir le transhumant. Sous un nombre de dents et de pieds trop grand, la prairje so ruine, comme nous l'avons vu: le pre se réduit dannee en annee, et ne peut plus bientot porter meme le quart des populations ovines qu'il recevait primitivement sans trop de peine. Il en résulte que, si la transhumance n'élait pas menacéc de mine au licu 
même d'origine de son mouton, par suite de la mise en valeur agricole et industrielle des páturages provençaux qu'il parcourait en hiver, il lui faudrait, en été, essaimer dans des montagnes de plus en plus étendues et nombreuses, dont elle accélérerait la ruine d'autant plus promptement que les chemins de fer se sont mis depuis plusieurs années à transporter ces envahisseurs d'un nouveau genre et a les deverser sur une aire de plus en plus agrandie.

Nous avons donc le devoir de combattre la transhumance partout où elle se manifeste.

Ce n'est pas là, quion le remarque bien, proscrire le mouton. C'esl en proscrire labus et le mauvais emploi, ce qui n'est pas la meme chose. Le mouton est utile par sa laine, sa chair, son laitage et son engrais; mais il faut lélever là où il est bienfaisant, les prés salés des bords de la mer, les plaines de la Champagne el du Berry, etc., etc., sont des terrains qui lui sont propices, et où il peut pulluler avanlageusement. Il ne sagit pas non plus de le proscrire completement de la montagne, il y est utile au montagnard comme nous lavons vu, il nous faul ajouter qu'il y a des pâturages qui ne sont accessibles qu'à lui seul. Dans celle mesure, il est bienfaisant, qu'il reste donc.

Il faudra montrer alt montagnard de quel avantage peut étro pour lui l'exploitation du gros bétail, surtout si, surmontant ce que son individualisme à de trop farouche, il sait associer ses capilaux pour celte exploitation. Troupeaux, étables et montagnes, pourvus et gérés à frais communs, fruitières exploitées dans le même esprit... elc., etc... avec toutes les améliorations de détail que cet agencement emporle avec lui.

Les commencements seront difficiles, les premiers pas seront courls ef lents, mais il n'est pas douteux que les résultats favorables les couronnant, lexemple ne fasse ensuite accélérer la rénovation que nous espérons.

Entre temps, il y aura lieu de leur montrer les meilleures méthodes de cultures de leurs prés, les avantages des irrigations, jadis si prospères dans nos montagnes el peu à jeu tombees clans loubli, etc., etc.

Vous serez d'autant mieux fondés à inciter les montagnards, à agir dans ce sens, que la transhumance disparaitra plus vite. Or, parmi les facteurs de sa rélrogradation, il y en a un, et non des moindres, qui est dans vos mains, je veux parler de laménagement des chutes d'eau. Partout où cela sera possible et équitable, intéressez puissamment les communes a la réalisation de la chute convoitee ; le versement que vous ferez alors d'une manière ou d'une autro, daus la caisse du municipe, y prendra souvent avec avantage la place qu'y tenaient les contributions des bergers étrangers el, spontanément, vos montagnards les ecarteront, car il ne faudrail pas croire qu'ils les roient venir chez eux sans dépit. De ces faits, jai pius que la conviction, lassurance, car nous sommes ici plusieurs qui les avons contrólés par la pratique. Apres le pré. le bois.

11 importe d'enrayer les destructions, jes défrichements inconsidérés, les labours routiniers el rujneux. Il laut démontrer au montagnard qu'il aura du ble, de l'avoine, du seigle meilleur et a meilleur compte au marche, et quil ne laut jamais déboiser les penles el que si, par exception, il déboise et laboure un replat, ccst pour $y$ faire une prairie, et non un labour annuel non plus ru'une culture sarclée.

Lhomme considère souvent lépargne conme un profif individuel, c'est une vue restreinte qui conslitue ainsi une erreur grave : léparqne est une nécessité sociale. Nos codes lindiquent un peu trop sommairemont du reste quand ils partent de la gestion du bon père de famille. Cetle verité il faut la bien meltre en lumiere et bien convaincre le prochain de son excellence. Quand chacun saura trouver en lui-meme la force de praliquer raisonnablement toules les epargnes, le fameux droil $\dot{a}$ labus qui cause tant de soucis a l'ecole interventionniste, disparaitra tout seul, et cet accouplement de mots ne cachera de sens pour personne, comme il est déjà, du reste, pour ceux qui savent aller au fond des choses. Il faut surtout créer des habitudes, des mœurs, en ces matières. Une loi coercitive qui prétendrait contraindre serait inefficace, il faut que la conviction vienne de l'homme lui-mème.

Et cotte épargne je ne crois pas avoir besoin de le dire, c'est non pas aux biens privés seuls qu'elle doil s'appliquer, mais aux biens publics aussi. Quand les promeneurs s'entendent pour respecler les arbres et les pelouses d'une promenade publique, ou il n'y a pas de gardien, ou les tableaux d'un muscée dans le mème cas, ils pratiquent déjà cette épargne. C'est l'extension de ces habitudes qu'il faut poursuivre.

Il faut faire comprendre aux propriétaires que leur intérêt bicn entendu consiste a jouir de la rente de leurs bois et a conserver le capital qu'ils conslituent.

Partout où elle est possible la production du bois d'œuvre doit être prélérée à celle des essences destinées aux usages chimiques ou au chauflage; a égalité de volume, sa valeur est souvent bien plus du double de celle du bois grossier.

Les forets, ainsi comprises, constituent un placement très rémunérateur; elles le seront d'autant plus que le flsc se montrera plus équitable et n'accablera pas d'impots excessif le sol portant des arbres.

De préférence ce sont les forêts des pentes et celles des berges des torrents qui doivent retenir l'attention encore que toutes, partout où elles sont situées, doivent la solliciter; mais c'est le plus urgent.

Il y a lieu aussi de poursuivre la constitulion de forèts dans les terres de plaines incultes et délaissées par la charrue : l'exemple des Landes de Gascogne et des dunes du Pas-rle-Calais, est des plus instructifs, el il montre que la richesse forestière en suscite bien d'autres derrière elle.

Quand toul cela sera fait, on pourra voir a creer des barrages de retenue, ils seront économiques et efficaces :

Cofle ourre vous semble immense! Elle l'est, mais si les bonnes volontés s'associent, elle sera facile à mener à bonne fin.

C'est seulement vers 1840 que Surell, ingénieur des ponts et chaussées dans les Hautes-Alpes, a jeté le premier cri d'alarme, et la déforestation avait été commencée par les Ro. mains ! Il fallu 30 ans pour qu'un continuateur lui vint, Cézanne, puis d'ébranlement donné, le mouvement s'accéléra, dans ces trente dernières années, surlout grâce aux efforts des fonctionnaires des eaux et forets. Depuis cinc ans, l'initiative privée s'est réveillée; de nombreuses sociétés se sont fondées qui, dans des vues differentes, font converger leurs efforts sur ce but.

Il $y$ en a plusieurs ici, mais une surtout es! plus intéressante que les aulres, al point de vue qui nous reticnt parce qu'elle sévertue à donner des leçons de choses el à y intéresser le monde industriel, et ses débuts, dans les deux sens sont du meilleur augure. Elle a trouvé un terrain de pâture en haute montagne, à interdire a la transhumance; elle y est arrivée pour une somme relativement modique el une année a suffi pour montrer qu'un pré peut se refaire tout seul quand on ne labandonne pas à une dépaissance abusive et déréglée.

Elle a trouvé des adeptes dévoués et généreux dans les industriels les plus distingués qui, imbus de loutes les idées que je viens de vous développer, ne craignent pals de prodiguer leur lemps el leur argent pour en amener la réussite matérielle.

Elle a trouvé aussi des collaborateurs dévoués dans la monlagne mème, et la chose vaut d'etre dite parce qu'on fait trop facilement au montagnard la réputation d'être irréductible sur les sujets. Non, il n'est pas irréduclible, mais it est pauvre, très pauvre, et il craint l'appareil coercitif du fonctionnarisme gouvernemental. Les formalites excitent sa defiance, car il les considère comme le prélude des vexalions. A-t-il donc tort? Vous savez déja vous qui m'avez écouté jusqu'ici, que je ne le condamne pas. 
Au lieu done domployer la naniene forte, de parler de survellance jalouse, de gardiens, de controleurs, nous avons prófere nous adressor à léguite et a la raison des gens. Vous arons passé des conventions précises, nous navons tracassé personne pour leü extevition et le résullat a depassé notre attente. Dabord, nous avms trouvé des gens Cort raisonnables aved lespucls nous mos sommes entendn. ensuile ayant bommenot mos faraux oh ! bien simple, ils ont consisté a ne rien faire, quempocher 300 provençax de tonche notre pró). co unc annóc particulicrment sèche. nous arons oblenu une reconstitution du palurage, telle que los gens du pays ont pu voir de lherbe dans les parlies ou its ben avaient jamais vu, mome par les antoes humides quand les moulons provençaux y fequentaient ! Io renseignement m'a éte apporlé arant-hier mino, il es nolve première récompense. Si nous avons bante les noubus provencau, nous navons pas tarté ceux des habilants its sont en petit nombre el leur presence sur le pré, riduile a ce petil tronfotu, ne peal elre que saluaire pour lo pro el pour ax. l.e rendenent des uns el cles aulres ina en saméliorant el ce sera le promier béncfice que les monlagnands recuedie. ront do la confiance cilits ont eu on mos ilces.

Ils en ont me atule. Sans doute la bisce communale se

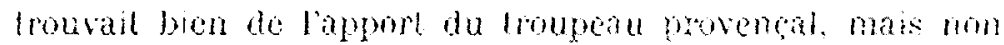
pas les proprietis particulieles, au bulument desquelles virait le lroupeau dans sa marcho vers son calivace. Cellesai avitient gandement a soufirir.

El puis les contestations entre propretnires ot bergers au sujel des degals commis par les betes des uns el des aulres. d lomui davoir des drangers chez soi. Toules rassons ifui militent en faveur de notre action.

Les transbunants étaent quelque olose comme los grandes compagnies de routiers du moyen aser lit ou ils avaient jasse il n'y avait pius rien; les chemins de nonlagne sonl bons tant que les tronpleax de provence n'y ont pas passe. rnsuite ils sont ruines et jl but les refare. Celte circonstance est de nature a interesser toutes les societes de chemins de ler. lourisles, asconsionnistes, elc, fui, a frais commun. font tracer des sentiers dans les monlagnes. Il laul quelles sa-

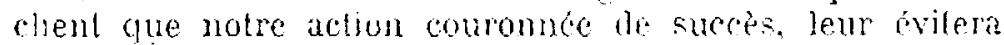
de lourdes charese pócuniaires.

$11 \mathrm{y}$ a done un réveil montrant que notre érergic nalive reprend conscione dellomone je devais vous lo diro et vols signaler res exemples: jajoulerai que si los jeunes lommes, qui se vouent comme vous aux dudes soientifilues beves. veutent bien nous apmorter jeur contingent defforts et dardeur, le resultal favorable ne me semble pas douteux.

Maintenont vous ai-je toul dit ? Non, hen sùr. Jans une esquisse lapide comme celle-ci on me pout dessimer quat sand ot gros traits, el nombre de detals, mène des détails importanls, doivent ètre laissés de coté Amsi, ai-je dú faire. mais je serais bien pave de ma peine, si, avec le peu que je rous ai dit, j’avais éte assez persuasif pour vous convincre quil $y$ avait da pour vous, dans lavenir, une bunde jeine d'utilite el diattrails.

Ce n'est pas en dilettanti que je vous conve a la laire, son agrement ne doil pas vous en acher lutitile, le devoir pour vous.

Ce mot vous paraitra peut-être bien gros aujourdhui, je men inquiète pou, la vie vous montrera assez combien jai mison!

Jajonterai que les moyeus de vous hrer à celte flude sont tous les jours plus multiplies et d'emploi plus tacile. II y a une très abondante littérature sur la question el clle saccroit tous les jours dexcellentes conlributions; il $y$ a de nombreux congres auxquels il faudra prendre part, cest li un relerendum de haule valeur; il y a les socieles d'etudes bénévoles auxquelles il faut vous inscrire et aux études desquelles vous avez le devoir de participer. Enfin, les moyens de communication perfectionnés rendent facile, non seule- ment la documentation livresque, mais celle des faits, la conviction de visu.

Vous vous souviendrez que ce nes pas pour sni seul yuin fitudie cue cest pour les autres. Vous vous devez a ros

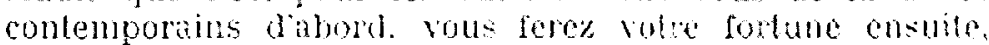
par suleroil, si voll pouvez, hials votre premior dewis. cest de vivre pond les antres. le contentement de rous. mente sera volve premete reomichse.

Commandant Alomentixn. ingenieme,

Ancien eleve de" Peole Polytechnique.

\section{Cipeulaire du 20 octobre 1906, concernant les instructions relatives à l'emploi du béton armé}

(Suite)

Les explications gui suivent ont pour objet de preciser is sens es la portée des instructions précédentes.

\section{1. - Données à admettre dans la préparation des projets.}

\section{A. Surcharges.}

Antictes 1, 2, 3. - De ces trois articies, les deux premiers se justifient d'eux-mîmes.

L.e troisieme, qui prescrit que les ouvrages qu'il vise seron calculés en vue des plus grandes surcharges quils aurom a supporter en service, semble inutilc, puisque tout ouvrage doit circ établi, et par conséquent calculé en vue de sa destination. C'est bien ie qui a lieu pour les ouvrages mélalliques ou aures qui ont précéde le ciment arme. On les calcule en vue des charges effecives les plus grantes auxquelles on prevoit qu'ils pourront être soumis, avec un coefficient de securité convenable, cest-à-dise de façon telle que, sous lefiet de ces charges, les forces élastiques natieignentqu'une fraction déterminée de celles qui seraient capables de produire la rupture.

pour les constructions ell béton armé, certains spécialistes preconisent une autre marche. Elle consisterai, non pas a chercher les forces elastiques décrminées par les surcharges effectives, mais a chercher dans quelle proportion il faudrait amplifier tictivement ces surcharges pour provoquer li rupture, et cest le coefficient damplitication qui serait, en ce cas, le coelficien de sécurité.

Cetre procédure, qui peut avoir son intécê, semble pourant ne pas devoir offrir de suffisantes garanties, parie que jamais un ouvrage ne périt par amplificarion proportionnelle des charges quil a a supporter. La chute dun ouvrage arrive soit par une cause accidentelle, soit par quelque mal interne dont le developpement tinit par ĉtre fatal.

Dans ces conditions, il semble convenable de calculer les ouvrages en béton armé comme les autres, pour les charges effectives les plus défavorables quils pourront avoir à supporter, et avec des coefficients de sécurite suffisants pour que ces charges ne puissent, à aucun degré, les meitre en danger.

Ces calculs sont oblipatoires. Mais, si les ingenieurs trouvent uile d'y joindre des calculs établis dans l'hyporhese de majorations des charges réelles, afin de se rendre compte des charges virtuelles qui provoqueraient la ruplure, ils sont libres de le faire, et d'exposer les conséquences qu'ils croiront pouvoir cn tirer.

\section{B. Limites de travail et de fatigue.}

Art. 4. - La limite de fatigue a la compression, fixé aux 28 centiemes de la résistance a l'écrasement du béton non armé. apres go jours de prise, est notablement plus élevée que celle généralement admise par les réglements ćurangers. Les chiffres resultant de ces derniers réglements conduiraient plutó à admetre, comme limite de fatigue à la compression d'un béton armć, le quart de la résistance à l'écrasement du béton similaire non armé, après 28 jours de prise. 